\title{
How the brain recognizes meaningful objects
}

\author{
ALBERTA STEINMAN GILINSKY \\ University of Bridgeport, Bridgeport, Connecticut
}

\begin{abstract}
Two neuropsychological approaches provide converging evidence for the neuronal basis of perceptual constancy of object recognition. Experimental studies of human patients with brain damage have found selective impairment of the ability to recognize particular categories of objects-faces, inanimate objects, bodily parts, and so forth, revealing mutually dissociable deficits. Visual cells in the monkey's temporal cortex have been found to be selectively sensitive to faces, head orientation, and direction of eye gaze. Results suggest that recognition of one type of object proceeds by independent high-level analysis of several restricted views of a given object processed at an earlier postsensory stage of perceptual categorization.
\end{abstract}

The idea that mental acts, perceptions, images, or concepts could be reducible to single neurons has seemed to some people to be outrageous, or at least highly implausible. Much of the resistance has come from people who hold the traditional view that mental phenomena and physical substances belong to entirely different realms. Some opposition stems from the apparently vast distance between the widely shared model of the nervous system as a fixed hierarchical structure, its hardware laid down under strict genetic control, and the remarkable flexibility and adaptability of human beings in an ever changing environment. Indeed, if the brain is innately determined and "hard-wired," how is learning possible?

Evolution has found a solution to this dilemma by constructing a heterarchically organized brain that is governed by a selecting agency. The striking feature of the brain as a selective system is the capacity of sensory stimulation by the environment to change the morphology of nervous connections and, by their own functional activity, to produce learning and memory. For example, an absence of normal visual stimulation during an early critical period in the life of an animal leads to altered development of the visual cortex. If a kitten is deprived of vision through one eye or is exposed to vertical but not to horizontal lines at a crucial stage of development, an actual shrinking or death of sensorially deprived fibers occurs, and their place is taken by successfully competing cells. Such anatomical variability provides a preexisting basis for the competitive selection during perceptual experience of those neurons that respond to a given sensory input.

\section{OVERVIEW OF COGNON THEORY}

For any behavior (e.g., stopping at a red traffic light), there must be at least one neuron that decides, on the basis of activity in the receptors and other neurons, whether

The author's mailing address is: Department of Psychology, University of Bridgeport, Bridgeport, CT 06601. to initiate that behavior. There may be as many single neurons of this sort as there are coherent behavior patterns and identifiable stimulus objects. The new neurobiology suggests that a neuronal representation may well underlie every one of the billions of things that you can, if you pay attention, recognize as an identifiable object or event.

The human brain has billions of neurons preprogrammed by epigenetically selected receptors as ready to lock on to this or that perceptual event, elicited by a biologically important object, as the frog's eye is preprogrammed to lock on to the movement of a fly. A pattern of incoming sensory impulses that finds its target in an actively expectant brain cell will facilitate recognition. I call these target brain cells, potentially recipient object detectors, cognitive neurons or cognons. Physiologically, the cognon is an object-recognition mechanism; psychologically, it is a mental representation. Thus, the cognon links the unit of cognition, the mental act or object, to the unit of biology, the neuron (see Gilinsky, 1984).

Every central representation that furnishes for perception the recognition of a meaningful object in response to the familiar external stimulus is formed through individual experience. Recognition of an object is recognition. It starts with perceptual acquisition-the initial formation of a mental representation in response to a novel or unexpected stimulus that evokes an investigatory response-Pavlov's "orientation reflex." This autonomic targeting reflex prepares the organism to identify the stimulus and to take whatever action may be needed to deal with it. If the new stimulus elicits an appetitive or aversive reaction, its perception will be reinforced and strengthen the corresponding internal representation.

Once established, cognons can take part in acquiring new associations both within and across modalities. Thus, cognons provide the essential building blocks for the construction of an orderly internal representation-a smallscale model-of the external world. The internalized model will necessarily be unique to the degree that every individual experiences a unique set of perceptual objects and events. 


\section{NEUROPSYCHOLOGICAL EVIDENCE}

My thesis is supported by two different lines of experimental investigation: (1) neuropsychological studies of human patients with localized brain lesions, and (2) microelectrode recordings from single neurons in the cortex of alert, behaving monkeys.

\section{Brain-Injured Humans}

The analysis of deficits in patients with brain injury reveals specific disturbances of recognition that result from damage to circumscribed areas of the brain. Disorders of recognition that clearly are not due to sensory impairment-agnosias-provide compelling evidence of multiple dissociations of syndromes. Thus, visual agnosias for objects, colors, topography, parts of the body, letters, numbers, and even handwriting, have all been identified as multiply dissociable deficits. The specificity and selectivity of these agnosias is striking. Prosopagnosia, the loss of the ability to recognize faces, is a dramatic example. These patients fail to identify close friends, family members, or even their own faces in the mirror, although they have no difficulty in recognizing other patterns or objects by visual inspection.

The ability to recognize faces is of particular interest. Even very young infants respond differentially to facial patterns. Perhaps no other object in the world is quite as important to us as is the human face. Since restricted brain damage in human patients can selectively impair recognition of faces while leaving the perception of other objects undisturbed, we may ask whether we can find specialized mechanisms in animals for selective responses to faces.

\section{Single-Cell Studies}

The most recent and telling evidence is from macaque monkeys that are unanesthetized and totally awake. Investigators in two British psychological laboratories, Rolls (Perrett \& Rolls, 1983; Perrett, Rolls, \& Caan, 1982) at the University of Oxford, and Perrett and his group (Perrett et al., 1984, 1985) at the University of St. Andrews in Scotland, recently reported populations of face-selective neurons in the temporal cortex of monkeys. These neurons are in the superior temporal sulcus that receives projections from the inferior temporal visual cortex. The major findings follow:

1. Face-selective Cells. The responses of 182 cells (about $77 \%$ ) of those in the superior temporal sulcus were responsive to the sight of the face or head but not to other objects. Most cells were sensitive to the orientation of the head with different views maximally exciting different classes of cell.

2. Face Identity. A substantial proportion of cells was sensitive to face identity, defined as the preferential response of a cell to one particular face over another. Cells that were highly selective for particular individuals familiar to the monkey maintained their selectivity over a wide variety of viewing conditions. One cell that responded to the face of one experimenter much more than to the face of another generalized across changing facial expressions, orientations, distance, and size. Another cell responded to a preferred face when presented full face only, but irrespective of the placement of a strong light that illuminated the face from the side, back, top, or below.

3. Basis for Face Constancy. Did cells respond to particular features or to face identity on the basis of its unique configuration? By presenting restricted regions of the face in isolation (eyes, lower mouth part, etc.) the answer was found to depend on the given cell. Thus, one cell was selectively responsive to certain features or isolated regions of a preferred face, and another cell was highly responsive to the sight of a preferred face in its entirety, rather than to any specific part.

4. Comparisons with Human Responses to the Same Stimuli. Conditions that increased latencies of response in monkeys (inversion, photographic negatives, filtered images with low spatial frequencies or color removed) were found similarly to lengthen human reaction time to faces seen under more usual viewing conditions.

5. Cortical Organization. Cells of a similar type were found to be grouped together both vertically through the cortex and horizontally in columns extending $1 / 2-2 \mathrm{~mm}$ across the surface of the cortex - a finding consistent with mounting physiological evidence that the cortex is organized in partially isolated modular units of categorically specific cell types.

\section{DISCUSSION}

The monkey data support the concept of single cells (cognons) as object detectors. The responses of these cells show selective recognition for face identity independent of changes in viewing conditions and facial expression. Together with the responses of five classes of viewselective cells, the findings suggest that object recognition proceeds in two distinct stages: a first stage in which cells specialized for each of the five possible views of the face or head pool their data for a particular individual face, and a second stage in which face-identity cells generalize across views as the object rotates relative to the viewer. The end result is a viewer-centered (monkey-centered) face cognon.

Warrington and associates have presented evidence for a comparable two-stage model for the categorical specificity of two postsensory systems in the human cerebral cortex that is remarkably consistent with the monkey single-cell data (Warrington, 1981, 1982, 1985; Warrington \& Shallice, 1984; Warrington \& Taylor, 1978) found that disorders of object recognition, such as the inability to identify familiar objects from fragmented or unusual viewpoints, were associated with cerebral lesions of the right posterior hemisphere. They suggested that the right posterior syndrome resulted from faulty perceptual categorization, a first postsensory categorical stage in object recognition. In contrast, patients with left hemisphere 
lesions were able to perform these perceptual tests but were unable to solve semantic tests that required matching physically dissimilar objects by functional identity, independently of their physical similarity to a third object (e.g., an open and a closed umbrella vs. a walking stick). The performance of both right- and left-lesion groups showed deficits on this functional or semantic categorization task. It seems that accurate semantic categorization requires preexisting perceptual categorization, an earlier level of analysis demanding intact right hemisphere function.

The two lines of neuropsychological research, human and monkey, converge to demonstrate that object recognition is not a diffusely distributed function of the entire cerebrum, indivisible into subfunctions, but rather is a composite of many different brain operations, relatively independent of one another.

With regard to face recognition, note that a face can be encoded in many different ways. A fundamental distinction divides the categorization of faces into higher order variables, such as individual identity, species, age, sex, and emotional state, that may be considered as functional equivalence classes. At a more primitive level, there are a number of perceptual categories based on variables that affect the appearance of a face or its recognizability as a pattern. These variables include position in the visual field, orientation, color, illumination, and so forth-the classical factors involved in studies of object constancy. To code for functional identity or other higher order variables, the underlying brain mechanisms need to be able to generalize over each of the perceptual variables and other incidental attributes, such as hairstyle.

In the monkey studies, the observed separation of cell classes in terms of their modular cortical organization and their selective responses to particular views of the face stimulus, together with the existence of other face-identity cells selective for individual faces irrespective of orientation, parallels Warrington's (1985) distinction between a primitive first stage in perceptual categorization and a second higher order stage of object recognition that requires both generalization and differentiation. Such a functional distinction, based on anatomically verifiable criteria, suggests a fruitful direction for further research.

\section{REFERENCES}

Gilinsky, A. S. (1984). Mind and brain. New York: Praeger.

Perrett, D. I., \& Rolls, E. T. (1983). Neural mechanisms underlying the visual analysis of faces. In J. P. Ewert, R. R. Caprinica, \& D. J. Ingle (Eds.), Advances in vertebrate neuroethology. New York: Plenum.

Perrett, D. I., Rolls, E. T., \& CAAN, W. (1982). Visual neurones responsive to faces in the monkey temporal cortex. Experimental Brain Research, 47, 329-342.

Perrett, D. I., Smith, P. A. J., Mistlin, A. J., Head, A. S., MilNER, A. D., \& JEEVES, M. A. (1984). Neurones responsive to faces in the temporal cortex: Studies of functional organization, sensitivity to identity and relation to perception. Human Neurobiology, 3, 197-208.

Perrett, D. I., Smith, P. A. J., Potter, D. D., Mistlin, A. J., Head, A. S., Milner, A. D., \& Jeeves, M. A. (1985). Visual cells in the temporal cortex sensitive to face view and gaze direction. Proceedings of the Royal Society (London), B223, 293-317.

WARrington, E. K. (1981). Neuropsychological studies of verbal semantic systems. Philosophical Transactions of the Royal Society (London), B295, 411-423.

WARRINGTON, E. K. (1982). Neuropsychological studies of object recognition. Philosophical Transactions of the Royal Society (London), B298, 15-33.

WARRINGTON, E. K. (1985). Agnosia: The impairment of object recognition. In J. A. M. Frederiks (Ed.), Handbook of Clinical Neurology, Vol. 1(45): Clinical Neuropsychology. Amsterdam: Elsevier.

Warrington, E. K., \& Shallice, T. A. (1984). Category-specific semantic impairments. Brain, 107, 829-854.

WARrington, E. K., \& TAYLOR, A. M. (1978). Two categorical stages of object recognition. Perception, 7, 695-705.

(Manuscript received for publication December 5, 1985.) 